Symmetries for exact solutions to the nonlinear Schrödinger equation

This article has been downloaded from IOPscience. Please scroll down to see the full text article.

2010 J. Phys. A: Math. Theor. 43025202

(http://iopscience.iop.org/1751-8121/43/2/025202)

The Table of Contents and more related content is available

Download details:

IP Address: 192.167.137.10

The article was downloaded on 11/12/2009 at 08:09

Please note that terms and conditions apply. 


\title{
Symmetries for exact solutions to the nonlinear Schrödinger equation
}

\author{
Tuncay Aktosun ${ }^{1}$, Theresa Busse ${ }^{1}$, Francesco Demontis ${ }^{2}$ and \\ Cornelis van der $\mathrm{Mee}^{2}$ \\ 1 Department of Mathematics, University of Texas at Arlington, Arlington, TX 76019-0408, USA \\ 2 Dipartimento di Matematica e Informatica, Università di Cagliari, Viale Merello 92, \\ 09123 Cagliari, Italy \\ E-mail: aktosun@uta.edu
}

Received 7 September 2009, in final form 30 October 2009

Published 10 December 2009

Online at stacks.iop.org/JPhysA/43/025202

\begin{abstract}
A certain symmetry is exploited in expressing exact solutions to the focusing nonlinear Schrödinger equation in terms of a triplet of constant matrices. Consequently, for any number of bound states with any number of multiplicities the corresponding soliton solutions are explicitly written in a compact form in terms of a matrix triplet. Conversely, from such a soliton solution the corresponding transmission coefficients, bound-state poles, bound-state norming constants and Jost solutions for the associated Zakharov-Shabat system are evaluated explicitly. These results also hold for the matrix nonlinear Schrödinger equation of any matrix size.
\end{abstract}

PACS numbers: $02.30 . \mathrm{Ik}, 05.45 . \mathrm{Yv}, 47.35 . \mathrm{Fg}$

Mathematics Subject Classification: 37K15, 35Q51, 35Q55

\section{Introduction}

Consider the focusing cubic nonlinear Schrödinger (NLS) equation

$$
\mathrm{i} u_{t}+u_{x x}+2|u|^{2} u=0
$$

where the subscripts denote appropriate partial derivatives. It arises in applications as diverse as wave propagation in nonlinear media [15], surface waves on sufficiently deep waters $[14,15]$ and signal propagation in optical fibers [10-12]. Its initial-value problem is known to be solvable by the inverse scattering transform method [1, 2, 13, 15]. In other words, certain solutions to (1.1) can be viewed as a potential in the Zakharov-Shabat system

$$
\frac{\mathrm{d} \varphi(\lambda, x, t)}{\mathrm{d} x}=\left[\begin{array}{cc}
-\mathrm{i} \lambda & u(x, t) \\
-u(x, t)^{*} & \mathrm{i} \lambda
\end{array}\right] \varphi(\lambda, x, t)
$$

where an asterisk is used to denote complex conjugation, and $u(x, t)$ can be recovered from $u(x, 0)$ with the help of the scattering data sets for (1.2) at $t=0$ and at time $t$. 
Exact solutions to nonlinear partial differential equations are of great interest. Such solutions may be helpful to better understand the corresponding nonlinearity, and they may also be useful in producing testing means to determine accuracy of numerical methods for solving nonlinear partial differential equations. This paper is related to exact solutions to $(1.1)$.

In previous papers $[3,6-8]$ we presented a method to construct exact solutions to (1.1) that are globally analytic on the entire $x t$-plane and decay exponentially as $x \rightarrow \pm \infty$ at each fixed $t \in \mathbf{R}$. This has been achieved by using a matrix triplet $(A, B, C)$, where all eigenvalues of $A$ have positive real parts. A similar method was applied to the Korteweg-de Vries equation on the half-line [4]. The same method is also applicable to various other nonlinear partial differential equations that are integrable by the inverse scattering transform with the help of a Marchenko integral equation.

In this paper we analyze the method of [3] when the eigenvalues of the matrix $A$ in the triplet $(A, B, C)$ do not all have positive real parts. It is already known that if one or more eigenvalues of $A$ are purely imaginary, the corresponding scattering coefficients for (1.2) contain discontinuities at some real values of $\lambda$ and hence the corresponding $u(x, t)$ cannot be analytic on the entire $x t$-plane. Furthermore, it is already known that for soliton solutions to (1.1), the corresponding transmission coefficients for (1.2) must have a pole and a zero appearing as a pair located symmetrically with respect to the real axis, which implies that a pair (or more pairs) of eigenvalues of $A$ cannot be located symmetrically with respect to the imaginary axis. Thus, in our paper we mainly concentrate on the case where eigenvalues of $A$ may occur anywhere on the complex plane, but no eigenvalues of $A$ are on the imaginary axis and no pairs of eigenvalues are located symmetrically with respect to the imaginary axis. For such triplets $(A, B, C)$ we show that there is an equivalent triplet $(\tilde{A}, \tilde{B}, \tilde{C})$ yielding the same solution $u(x, t)$ to $(1.1)$, where all eigenvalues of $\tilde{A}$ have positive real parts. The corresponding solutions $u(x, t)$ are then analytic on the entire $x t$-plane and they are soliton solutions with any number of poles in the corresponding transmission coefficients and with any multiplicities of such poles. For such triplets we also explicitly evaluate the corresponding transmission coefficients, bound-state norming constants and the corresponding Jost solutions to (1.2).

Our paper is organized as follows. In section 2 we present the preliminary material by providing an outline of the method of [3] and introduce exact solutions $u(x, t)$ to (1.1) constructed via a triplet $(A, B, C)$. In section 3 we exploit a certain symmetry in such exact solutions and show that some (or all) eigenvalues of $A$ can be chosen either on the right or on the left half complex plane without changing $u(x, t)$. In section 4 we show that such solutions are solitons with any number of poles in the corresponding transmission coefficients and with any multiplicities of such poles. We also explicitly evaluate the corresponding transmission coefficients and bound-state norming constants and the Jost solutions to (1.2). Finally, we conclude in section 5 with an explicit example, which was earlier studied as example 7.2 of [3]. Since one of the eigenvalues of $A$ in that example has a negative real part, we earlier conjectured that it might be a nonsoliton solution. Using our current results, we verify in section 5 that it is a two-soliton solution and we explicitly evaluate the corresponding transmission coefficients, the bound-state norming constants and the Jost solutions to (1.2).

We state the results of sections 2- 4 carefully so that they remain valid for the matrix NLS equation

$$
\mathrm{i} u_{t}+u_{x x}+2 u u^{\dagger} u=0
$$

where $u$ is now $m \times n$ matrix valued, the dagger denotes the matrix adjoint (matrix transpose and complex conjugate) and the associated Zakharov-Shabat system is given by 


$$
\frac{\mathrm{d} \varphi(\lambda, x, t)}{\mathrm{d} x}=\left[\begin{array}{cc}
-\mathrm{i} \lambda I_{m} & u(x, t) \\
-u(x, t)^{\dagger} & \mathrm{i} \lambda I_{n}
\end{array}\right] \varphi(\lambda, x, t),
$$

with $I_{n}$ denoting the $n \times n$ identity matrix.

\section{Preliminaries}

In this section we establish our notation and provide the preliminaries for certain exact solutions to the focusing NLS equation. Such exact solutions are expressed [3] in terms of a triplet of matrices $(A, B, C)$.

Consider any triplet $(A, B, C)$, where $A$ is a $p \times p$ (complex-valued) constant matrix, $B$ is a $p \times 1$ (complex-valued) constant matrix and $C$ is a $1 \times p$ (complex-valued) constant matrix. For short, we will refer to such a triplet as a triplet of size $p$. From such a triplet, construct the auxiliary $p \times p$ matrices $Q$ and $N$ by solving the respective Lyapunov equations

$$
\left\{\begin{array}{l}
Q A+A^{\dagger} Q=C^{\dagger} C \\
A N+N A^{\dagger}=B B^{\dagger}
\end{array}\right.
$$

Note that if $(Q, N)$ satisfies the system in (2.1), so does $\left(Q^{\dagger}, N^{\dagger}\right)$; hence, there is no loss of generality in assuming that the solution matrices $Q$ and $N$ to (2.1) are self-adjoint. Then form the $p \times p$ matrix $F(x, t)$ and the scalar quantity $u(x, t)$ as

$$
\begin{aligned}
& F(x, t):=\mathrm{e}^{2 A^{\dagger} x-4 \mathrm{i}\left(A^{\dagger}\right)^{2} t}+Q \mathrm{e}^{-2 A x-4 \mathrm{i} A^{2} t} N, \\
& u(x, t):=-2 B^{\dagger} F(x, t)^{-1} C^{\dagger} .
\end{aligned}
$$

Let us also define the $p \times p$ matrix $G(x, t)$ and the scalar quantity $v(x, t)$ as

$$
\begin{aligned}
& G(x, t):=\mathrm{e}^{-2 A x-4 \mathrm{i} A^{2} t}+N \mathrm{e}^{2 A^{\dagger} x-4 \mathrm{i}\left(A^{\dagger}\right)^{2} t} Q, \\
& v(x, t):=-2 C G(x, t)^{-1} B .
\end{aligned}
$$

Theorem 2.1. Given a triplet $(A, B, C)$ of size $p$, let $(Q, N)$ be a self-adjoint solution to the system in (2.1). Then $u(x, t)$ defined as in (2.3) satisfies the NLS equation (1.1) at any point on the xt-plane where $F(x, t)$ is invertible. Similarly, $v(x, t)$ defined in (2.5) satisfies (1.1) at any point on the $x t$-plane where $G(x, t)$ is invertible.

Proof. Let us only give the proof for $v$ because the proof for $u$ is similar. In fact, $v(x, t)$ turns into $u(-x, t)$ by replacing $(A, B, C)$ with $\left(A^{\dagger}, C^{\dagger}, B^{\dagger}\right)$, which can also be used for the proof related to $u(x, t)$. Let us drop the arguments of the functions involved and write (2.4) and its adjoint as

$$
G=\mathrm{e}^{-\beta}+N \mathrm{e}^{\beta^{\dagger}} Q, \quad G^{\dagger}=\mathrm{e}^{-\beta^{\dagger}}+Q \mathrm{e}^{\beta} N
$$

where we have defined

$$
\beta:=2 A x+4 \mathrm{i} A^{2} t
$$

Note that $v$ in (2.5) is well defined as long as $G^{-1}$ exists. Taking appropriate partial derivatives, from (2.5) and (2.6) we obtain

$$
\begin{aligned}
& \mathrm{i} v_{t}+v_{x x}+2 v v^{\dagger} v=-2 C G^{-1} P G^{-1} B, \\
& P:=-\mathrm{i} G_{t}-G_{x x}+2 G_{x} G^{-1} G_{x}+8 B B^{\dagger}\left(G^{\dagger}\right)^{-1} C^{\dagger} C .
\end{aligned}
$$


From (2.6) we get

$G_{t}=-4 \mathrm{i} A^{2} \mathrm{e}^{-\beta}-4 \mathrm{i} N\left(A^{\dagger}\right)^{2} \mathrm{e}^{\beta^{\dagger}} Q, \quad G_{x}=-2 A \mathrm{e}^{-\beta}+2 N A^{\dagger} \mathrm{e}^{\beta^{\dagger}} Q$,

$G_{x x}=4 A^{2} \mathrm{e}^{-\beta}+4 N\left(A^{\dagger}\right)^{2} \mathrm{e}^{\beta^{\dagger}} Q$,

$\mathrm{e}^{\beta^{\dagger}} Q G^{-1}=\left(G^{\dagger}\right)^{-1} Q \mathrm{e}^{\beta}, \quad \mathrm{e}^{\beta} N\left(G^{\dagger}\right)^{-1}=G^{-1} N \mathrm{e}^{\beta^{\dagger}}$.

With the help of (2.1), (2.6), and (2.9)-(2.11), one can verify that $P=0$, and hence the right-hand side of (2.8) is zero.

There are several questions that can be raised. For example, are the Lyapunov equations given in (2.1) solvable; if they are solvable, are they uniquely solvable? Are the matrices $F$ and $G$ defined in (2.2) and (2.4), respectively, invertible? The answers to these questions are affirmative under appropriate restrictions on the triplet $(A, B, C)$, as we will see.

Let us note that $u$ and $v$ defined in (2.3) and (2.5), respectively, are analytic functions of $x$ and $t$ at any point on the $x t$-plane as long as the matrices $F$ and $G$, respectively, are invertible at that point. This is because the entries of those matrices and hence also their determinants can be written as sums of products of sine, cosine, exponential and polynomial functions of linear combinations of $x$ and $t$.

Consider the scalar function $\Omega$ defined as

$$
\Omega(x):=C \mathrm{e}^{-A x} B \text {. }
$$

The right-hand side is called a matrix realization of $\Omega$ in terms of the triplet $(A, B, C)$. Without changing $\Omega(x)$, it is possible to increase the value of $p$ in the size of the triplet $(A, B, C)$ by padding the matrices $A, B, C$ with zeros or by modifying $A, B, C$ in some other fashion (cf [8], section 2.4). Conversely, it might also be possible to reduce the value of $p$ in the triplet $(A, B, C)$ so that the quantity $\Omega(x)$ will remain unchanged. The matrix realization in $(2.12)$ is said to be minimal if the value of $p$ in the triplet $(A, B, C)$ is the smallest and yet $\Omega(x)$ remains unchanged by the choice of $p$. The triplet $(A, B, C)$ is minimal if and only if [5] the intersections of the kernels of $C A^{j}$ and of the kernels of $B^{\dagger}\left(A^{\dagger}\right)^{j}$ for $j=0,1,2, \ldots$ are trivial, i.e.

$\left\{\xi \in \mathbf{C}^{p}: C A^{j} \xi=0\right.$ for $\left.j \geqslant 0\right\}=\{0\}=\left\{\eta \in \mathbf{C}^{p}: B^{\dagger}\left(A^{\dagger}\right)^{j} \eta=0\right.$ for $\left.j \geqslant 0\right\}$.

It is also known [5] that a triplet yielding a minimal realization in (2.12) is unique up to a similarity transformation $(A, B, C) \mapsto\left(E A E^{-1}, E B, C E^{-1}\right)$ for some unique matrix $E$.

The results in the next theorem are known [3], but they are collected here in a summarized form and a brief proof is included for the benefit of the reader.

Theorem 2.2. Assume that the triplet $(A, B, C)$ of size p corresponds to a minimal realization in (2.12) and that the eigenvalues of $A$ all have positive real parts. Then

(i) The Lyapunov equations in (2.1) are uniquely solvable.

(ii) The solutions $Q$ and $N$ are $p \times p$ self-adjoint matrices.

(iii) $Q$ and $N$ can be expressed in terms of the triplet $(A, B, C)$ as

$$
Q=\int_{0}^{\infty} \mathrm{d} s\left[C \mathrm{e}^{-A s}\right]^{\dagger}\left[C \mathrm{e}^{-A s}\right], \quad N=\int_{0}^{\infty} \mathrm{d} s\left[\mathrm{e}^{-A s} B\right]\left[\mathrm{e}^{-A s} B\right]^{\dagger} .
$$

(iv) $Q$ and $N$ are invertible matrices.

(v) Any square submatrix of $Q$ containing the $(1,1)$-entry or $(p, p)$-entry of $Q$ is invertible. Similarly, any square submatrix of $N$ containing the $(1,1)$-entry or $(p, p)$-entry of $N$ is invertible.

(vi) The $p \times p$ matrix quantities $F$ and $G$ defined in (2.2) and (2.4), respectively, are invertible at every point on the $x t$-plane. 
Proof. By introducing the parameter $\alpha$, let us write the first equation in (2.1) as

$$
-Q(\alpha I-A)+\left(\alpha I+A^{\dagger}\right) Q=C^{\dagger} C,
$$

or equivalently as

$$
-\left(\alpha I+A^{\dagger}\right)^{-1} Q+Q(\alpha I-A)^{-1}=\left(\alpha I+A^{\dagger}\right)^{-1} C^{\dagger} C(\alpha-A)^{-1},
$$

where $I$ is the $p \times p$ identity matrix. Since $A$ and $\left(-A^{\dagger}\right)$ have eigenvalues on the right and left complex half planes, respectively, we can integrate (2.15) along a simple and positively oriented contour $\gamma$ lying on the right half complex plane and enclosing all eigenvalues of $A$. Thus, we obtain $Q$ uniquely as

$$
Q=\frac{1}{2 \pi \mathrm{i}} \int_{\gamma} \mathrm{d} \alpha\left(\alpha I+A^{\dagger}\right)^{-1} C^{\dagger} C(\alpha I-A)^{-1} .
$$

Similarly, the solution to the second equation in (2.1) is unique and is obtained as

$$
N=\frac{1}{2 \pi \mathrm{i}} \int_{\gamma} \mathrm{d} \alpha(\alpha I-A)^{-1} B B^{\dagger}\left(\alpha I+A^{\dagger}\right)^{-1} .
$$

Thus, (i) is proved. From (2.1) it is seen that $\left(Q^{\dagger}, N^{\dagger}\right)$ is a solution to (2.1) whenever $(Q, N)$ is a solution and hence from the uniqueness of the solution we obtain (ii). From (2.14) we see that

$$
Q A+A^{\dagger} Q=-\int_{0}^{\infty} \mathrm{d} s \frac{\mathrm{d}}{\mathrm{d} s}\left[\mathrm{e}^{-A^{\dagger} s} C^{\dagger} C \mathrm{e}^{-A s}\right]=-\left.\mathrm{e}^{-A^{\dagger} s} C^{\dagger} C \mathrm{e}^{-A s}\right|_{s=0} ^{\infty}=C^{\dagger} C,
$$

where we have used the fact that all eigenvalues of $A$ have positive real parts. A similar argument for $N$ completes the proof of (iii). From their self-adjointness and positivity as seen from (2.14), it follows that all eigenvalues of $Q$ and $N$ are nonnegative. Moreover, (2.13) implies that zero is not an eigenvalue of $Q$ or $N$. Hence $Q$ and $N$ are invertible, proving (iv). The positivity of all eigenvalues also implies (v). The invertibility of $F$ follows from using theorem 4.2 of [3] in (2.2), and the proof of invertibility for $G$ is similar.

The results in the next theorem are useful in extracting the scattering data for (1.2) from the corresponding potential $u(x, t)$, which is also a solution to (1.1). For the benefit of the reader we state such results in a summarized and unified form. The proofs of these results are available in theorems 3.1 and 3.3 of [3], and hence they will not be given here.

Theorem 2.3. Assume that the triplet $(\tilde{A}, \tilde{B}, \tilde{C})$ of size p corresponds to a minimal realization in (2.12) and that the eigenvalues of $\tilde{A}$ all have positive real parts. Further, assume that $\tilde{A}$ has $m$ distinct eigenvalues $\alpha_{1}, \ldots, \alpha_{m}$ and the multiplicity of $\alpha_{j}$ is $n_{j}$. Then:

(i) There exists a unique triplet $(A, B, C)$, where $A$ is in a Jordan canonical form with each Jordan block containing a distinct eigenvalue and having -1 in the superdiagonal entries, and the entries of $B$ consist of zeros and ones. More specifically, we have

$$
\begin{aligned}
& A=\left[\begin{array}{cccc}
A_{1} & 0 & \cdots & 0 \\
0 & A_{2} & \cdots & 0 \\
\vdots & \vdots & \ddots & \vdots \\
0 & 0 & \cdots & A_{m}
\end{array}\right], \quad B=\left[\begin{array}{c}
B_{1} \\
B_{2} \\
\vdots \\
B_{m}
\end{array}\right], \quad C=\left[\begin{array}{llll}
C_{1} & C_{2} & \cdots & C_{m}
\end{array}\right] \text {, } \\
& A_{j}:=\left[\begin{array}{ccccc}
\alpha_{j} & -1 & 0 & \cdots & 0 \\
0 & \alpha_{j} & -1 & \cdots & 0 \\
0 & 0 & \alpha_{j} & \cdots & 0 \\
\vdots & \vdots & \vdots & \ddots & \vdots \\
0 & 0 & 0 & \cdots & \alpha_{j}
\end{array}\right], \quad B_{j}:=\left[\begin{array}{c}
0 \\
0 \\
0 \\
\vdots \\
1
\end{array}\right], \quad C_{j}:=\left[\begin{array}{lllll}
c_{j\left(n_{j}-1\right)} & \cdots & c_{j 1} & c_{j 0}
\end{array}\right]
\end{aligned}
$$


where $A_{j}$ has size $n_{j} \times n_{j}, B_{j}$ has size $n_{j} \times 1, C_{j}$ has size $1 \times n_{j}$ and the constants $c_{j\left(n_{j}-1\right)}$ are nonzero.

(ii) The triplet $(A, B, C)$ can be constructed from $(\tilde{A}, \tilde{B}, \tilde{C})$ via

$$
\tilde{A}=M A M^{-1}, \quad \tilde{B}=M S B, \quad C=\tilde{C} M S,
$$

where $M$ is a matrix whose columns are formed from the generalized eigenvectors of $(-\tilde{A})$ and $S$ is an upper triangular Toeplitz matrix commuting with $A$, is uniquely determined by $M$ and $\tilde{B}$, and has the form

$$
S=\left[\begin{array}{cccc}
S_{1} & 0 & \cdots & 0 \\
0 & S_{2} & \cdots & 0 \\
\vdots & \vdots & \ddots & \vdots \\
0 & 0 & \cdots & S_{m}
\end{array}\right], \quad S_{j}:=\left[\begin{array}{cccc}
\theta_{j n_{j}} & \theta_{j\left(n_{j}-1\right)} & \cdots & \theta_{j 1} \\
0 & \theta_{j n_{j}} & \cdots & \theta_{j 2} \\
\vdots & \vdots & \ddots & \vdots \\
0 & 0 & \cdots & \theta_{j n_{j}}
\end{array}\right]
$$

for some constants $\theta_{j s}$.

(iii) The triplets $(A, B, C)$ and $(\tilde{A}, \tilde{B}, \tilde{C})$ yield the same solution $u(x, t)$ to (1.1).

(iv) The complex constants $\left(i \alpha_{j}\right)$ correspond to the bound-state poles on the upper half complex plane of the transmission coefficients $T_{1}$ and $T_{\mathrm{r}}$ appearing in (4.2)-(4.5) of section 4.

(v) For each $j$, the complex constants $c_{j s}$ for $s=0,1, \ldots,\left(n_{j}-1\right)$ appearing in C correspond to the bound-state norming constants associated with the bound-state pole $\left(i \alpha_{j}\right)$ of the transmission coefficients.

\section{Symmetries for the NLS equation}

In this section we exploit a certain symmetry in (2.2) and show that without changing the value of the scalar $u(x, t)$ in (2.3) it is possible to transform the triplet $(A, B, C)$ in such a way that some or all eigenvalues of $A$ can be reflected from the right half complex plane to the left half complex plane. The same result holds for the scalar $v(x, t)$ given in (2.5); namely, it remains unchanged when the triplet $(A, B, C)$ is transformed so that some or all eigenvalues of $A$ are reflected with respect to the imaginary axis on the complex plane.

For repeated eigenvalues of $A$, the aforementioned transformation must be applied to all the multiplicities in such a way that after the transformation we should not have any eigenvalue pairs symmetrically located with respect to the imaginary axis of the complex plane. As mentioned in section 1, eigenvalue pairs symmetrically located with respect to the imaginary axis cannot yield soliton solutions to (1.1). For such pairs, it is already known [9] that (2.1) is not uniquely solvable.

Let us write (2.2) as

$$
F(x, t)=Q\left[\mathrm{e}^{-2 A x-4 A^{2} t}+Q^{-1} \mathrm{e}^{2 A^{\dagger} x-4 \mathrm{i}\left(A^{\dagger}\right)^{2} t} N^{-1}\right] N .
$$

Comparing (2.2) and (3.1), we next prove that $u(x, t)$ appearing in (2.3) remains invariant under the transformation

$$
(A, B, C, Q, N) \mapsto\left(-A^{\dagger},-N^{-1} B,-C Q^{-1},-Q^{-1},-N^{-1}\right),
$$

where all the eigenvalues of $A$ are reflected with respect to the imaginary axis on the complex plane as a result of $A \mapsto\left(-A^{\dagger}\right)$.

Theorem 3.1. Assume that the triplet $(A, B, C)$ corresponds to a minimal realization in (2.12) and that all eigenvalues of $A$ have positive real parts. Consider the transformation

$$
(A, B, C, Q, N, F, G, u, v) \mapsto(\tilde{A}, \tilde{B}, \tilde{C}, \tilde{Q}, \tilde{N}, \tilde{F}, \tilde{G}, \tilde{u}, \tilde{v}),
$$


where $(Q, N)$ corresponds to the unique solution to the Lyapunov system in (2.1), the quantities in $(F, G, u, v)$ are as in (2.2)-(2.5),

$\tilde{A}=-A^{\dagger}, \quad \tilde{B}=-N^{-1} B, \quad \tilde{C}=-C Q^{-1}, \quad \tilde{Q}=-Q^{-1}, \quad \tilde{N}=-N^{-1}$,

and $(\tilde{F}, \tilde{G}, \tilde{u}, \tilde{v})$ is as in (2.2)-(2.5) but by using $(\tilde{A}, \tilde{B}, \tilde{C}, \tilde{Q}, \tilde{N})$ instead of $(A, B, C, Q, N)$ on the right-hand sides. We then have the following:

(a) The quantities $F$ and $G$ are transformed as

$$
\tilde{F}=Q^{-1} F N^{-1}, \quad \tilde{G}=N^{-1} G Q^{-1} .
$$

(b) $\tilde{Q}$ and $\tilde{N}$ satisfy the respective Lyapunov equations

$$
\left\{\begin{array}{l}
\tilde{Q} \tilde{A}+\tilde{A}^{\dagger} \tilde{Q}=\tilde{C}^{\dagger} \tilde{C} \\
\tilde{A} \tilde{N}+\tilde{N} \tilde{A}^{\dagger}=\tilde{B} \tilde{B}^{\dagger}
\end{array}\right.
$$

(c) The matrices $\tilde{Q}$ and $\tilde{N}$ are self-adjoint and invertible.

(d) The matrices $\tilde{F}$ and $\tilde{G}$ are invertible at every point on the $x t$-plane.

(e) $\tilde{u}(x, t)=u(x, t)$ and $\tilde{v}(x, t)=v(x, t)$.

Proof. Using (3.3) in (2.2) and (2.4) we get (a). Using (2.1) and (3.3), it can directly be verified that (3.5) is satisfied, proving (b). Using (ii) and (iv) of theorem 2.2 in (3.3), it follows that (c) holds. The invertibility in (d) follows from (3.4) by using the invertibility of $Q$ and $N$ stated in (c) and the invertibility of $F$ and $G$ stated in (vi) of theorem 2.2. Finally, (e) can be proved by using (2.3) and (2.5) with the help of (3.3), the self-adjointness of $Q$ and $N$, and (3.4).

Next, we show that even if we reflect some of eigenvalues of $A$ from the right to the left half complex plane, theorem 3.1 remains valid by choosing the transformation in (3.2) appropriately. For this purpose, let us again start with a triplet $(A, B, C)$ of size $p$ and corresponding to a minimal realization in (2.12), where the eigenvalues of $A$ all have positive real parts. Without loss of any generality, let us partition $A, B, C$ as

$$
A=\left[\begin{array}{cc}
A_{1} & 0 \\
0 & A_{2}
\end{array}\right], \quad B=\left[\begin{array}{l}
B_{1} \\
B_{2}
\end{array}\right], \quad C=\left[\begin{array}{ll}
C_{1} & C_{2}
\end{array}\right],
$$

so that the $q \times q$ block diagonal matrix $A_{1}$ contains the eigenvalues that will remain unchanged and $A_{2}$ contains the eigenvalues that will be reflected with respect to the imaginary axis on the complex plane, the submatrices $B_{1}$ and $C_{1}$ have sizes $q \times 1$ and $1 \times q$, respectively, and hence $A_{2}, B_{2}, C_{2}$ have sizes $(p-q) \times(p-q),(p-q) \times 1,1 \times(p-q)$, respectively, for some integer $q$ not exceeding $p$. Let us write the corresponding respective solutions to (2.1) as

$$
Q=\left[\begin{array}{ll}
Q_{1} & Q_{2} \\
Q_{3} & Q_{4}
\end{array}\right], \quad N=\left[\begin{array}{ll}
N_{1} & N_{2} \\
N_{3} & N_{4}
\end{array}\right]
$$

where $Q_{1}$ and $N_{1}$ have sizes $q \times q, Q_{4}$ and $N_{4}$ have sizes $(p-q) \times(p-q)$, etc. Note that because of the self-adjointness of $Q$ and $N$ stated in theorem 2.2, we have

$$
Q_{1}^{\dagger}=Q_{1}, \quad Q_{2}^{\dagger}=Q_{3}, \quad Q_{4}^{\dagger}=Q_{4}, \quad N_{1}^{\dagger}=N_{1}, \quad N_{2}^{\dagger}=N_{3}, \quad N_{4}^{\dagger}=N_{4}
$$

Furthermore, from theorem 2.2 (v) it follows that $Q_{1}, Q_{4}, N_{1}$ and $N_{4}$ are all invertible.

Let us clarify our notational choice in (3.6) and emphasize that the partitioning in (3.6) is not the same partitioning used in (i) of theorem 2.3. 
Theorem 3.2. Assume that the triplet $(A, B, C)$ partitioned as in (3.6) corresponds to a minimal realization in (2.12) and that all eigenvalues of $A$ have positive real parts. Consider the transformation (3.2) with $(\tilde{A}, \tilde{B}, \tilde{C})$ having similar block representations as in (3.6), $(Q, N)$ as in (3.7) corresponding to the unique solution to the Lyapunov system in (2.2),

$\tilde{A}_{1}=A_{1}, \quad \tilde{A}_{2}=-A_{2}^{\dagger}, \quad \tilde{B}_{1}=B_{1}-N_{2} N_{4}^{-1} B_{2}, \quad \tilde{B}_{2}=-N_{4}^{-1} B_{2}$,

$\tilde{C}_{1}=C_{1}-C_{2} Q_{4}^{-1} Q_{3}, \quad \tilde{C}_{2}=-C_{2} Q_{4}^{-1}$,

and $(\tilde{Q}, \tilde{N})$ given as

$\tilde{Q}_{1}=Q_{1}-Q_{2} Q_{4}^{-1} Q_{3}, \quad \tilde{Q}_{2}=-Q_{2} Q_{4}^{-1}, \quad \tilde{Q}_{3}=-Q_{4}^{-1} Q_{3}, \quad \tilde{Q}_{4}=-Q_{4}^{-1}$,

$\tilde{N}_{1}=N_{1}-N_{2} N_{4}^{-1} N_{3}, \quad \tilde{N}_{2}=-N_{2} N_{4}^{-1}, \quad \tilde{N}_{3}=-N_{4}^{-1} N_{3}, \quad \tilde{N}_{4}=-N_{4}^{-1}$,

and $(\tilde{F}, \tilde{G}, \tilde{u}, \tilde{v})$ as in (2.2)-(2.5) but by using $(\tilde{A}, \tilde{B}, \tilde{C}, \tilde{Q}, \tilde{N})$ instead of $(A, B, C, Q, N)$ on the right-hand sides. We then have the following:

(a) The quantities $F$ and $G$ are transformed according to

$$
\begin{aligned}
& \tilde{F}=\left[\begin{array}{cc}
I & -Q_{2} Q_{4}^{-1} \\
0 & -Q_{4}^{-1}
\end{array}\right] F\left[\begin{array}{cc}
I & 0 \\
-N_{4}^{-1} N_{3} & -N_{4}^{-1}
\end{array}\right], \\
& \tilde{G}=\left[\begin{array}{cc}
I & -N_{2} N_{4}^{-1} \\
0 & -N_{4}^{-1}
\end{array}\right] G\left[\begin{array}{cc}
I & 0 \\
-Q_{4}^{-1} Q_{3} & -Q_{4}^{-1}
\end{array}\right] .
\end{aligned}
$$

(b) $\tilde{Q}$ and $\tilde{N}$ satisfy the respective Lyapunov equations in (3.5).

(c) The matrices $\tilde{Q}$ and $\tilde{N}$ are self-adjoint and invertible.

(d) The matrices $\tilde{F}$ and $\tilde{G}$ are invertible at every point on the $x t$-plane.

(e) $\tilde{u}(x, t)=u(x, t)$ and $\tilde{v}(x, t)=v(x, t)$.

Proof. Let us use $I$ to denote the identity matrix not necessarily having the same dimension in every appearance in the proof, but that dimension will be apparent to the reader. Note that (3.13) and (3.14) can be verified by using (3.8)-(3.12) in (2.2) and (2.4), which proves (a). The proof of (b) is by direct substitution in (3.5) and by using (2.1) and (3.6)-(3.12) and by noting that

$$
\begin{aligned}
& \tilde{B}=\left[\begin{array}{cc}
I & -N_{2} N_{4}^{-1} \\
0 & -N_{4}^{-1}
\end{array}\right] B=\left[\begin{array}{cc}
I & -N_{2} \\
0 & -N_{4}
\end{array}\right]^{-1} B, \\
& \tilde{C}=C\left[\begin{array}{cc}
I & 0 \\
-Q_{4}^{-1} Q_{3} & -Q_{4}^{-1}
\end{array}\right]=C\left[\begin{array}{cc}
I & 0 \\
-Q_{3} & -Q_{4}
\end{array}\right]^{-1},
\end{aligned}
$$

where the invertibility of $Q_{4}$ and $N_{4}$ is also used, which follows from theorem $2.2(\mathrm{v})$. The self-adjointness of $\tilde{Q}$ and $\tilde{N}$ follows from (3.7), (3.8), (3.11) and (3.12). The invertibility of $\tilde{Q}$ and $\tilde{N}$ can be seen from (3.11) and (3.12) by writing

$\tilde{Q}=\left[\begin{array}{cc}Q_{1} & Q_{2} \\ 0 & I\end{array}\right]\left[\begin{array}{cc}I & 0 \\ -Q_{4}^{-1} Q_{3} & -Q_{4}^{-1}\end{array}\right]=\left[\begin{array}{cc}Q_{1}^{-1} & -Q_{1}^{-1} Q_{2} \\ 0 & I\end{array}\right]^{-1}\left[\begin{array}{cc}I & 0 \\ -Q_{3} & -Q_{4}\end{array}\right]^{-1}$ 
and a similar expression for $\tilde{N}$ and the fact that $Q_{1}, Q_{4}, N_{1}, N_{4}$ are all invertible. Thus, (c) is proved. The invertibility in (d) follows from (3.13) and (3.14) by using the invertibility of $Q_{4}$ and $N_{4}$ stated in (v) of theorem 2.2 and the invertibility of $F$ and $G$ stated in (vi) of theorem 2.2. Finally, we evaluate $\tilde{F}(x, t)$ by using $(\tilde{A}, \tilde{B}, \tilde{C}, \tilde{Q}, \tilde{N})$ instead of $(A, B, C, Q, N)$ on the right-hand side of $(2.2)$ and evaluate $\tilde{u}(x, t)$ by using $(\tilde{B}, \tilde{F}, \tilde{C})$ instead of $(B, F, C)$ on the right-hand side of (2.3). With the help of (3.7)-(3.17), it is straightforward to show that $\tilde{u}(x, t)$ simplifies to $u(x, t)$; similarly, $\tilde{v}(x, t)$ simplifies to $v(x, t)$, completing the proof of (e).

Finally in this section, we show that $u$ and $v$ defined in (2.3) and (2.5), respectively, can be transformed into each other by transforming the triplet $(A, B, C)$ in a particular way.

Theorem 3.3. Assume that the triplet $(A, B, C)$ corresponds to a minimal realization in (2.12) and that all eigenvalues of $A$ have positive real parts. Consider the transformation (3.2) where $(Q, N)$ corresponds to the unique solution to the Lyapunov system in (2.1) and $(F, G, u, v)$ is as in (2.2)-(2.5),

$\tilde{A}=A, \quad \tilde{B}=Q^{-1} C^{\dagger}, \quad \tilde{C}=B^{\dagger} N^{-1}, \quad \tilde{Q}=N^{-1}, \quad \tilde{N}=Q^{-1}$,

and $(\tilde{F}, \tilde{G}, \tilde{u}, \tilde{v})$ is as in (2.2)-(2.5) but by using $(\tilde{A}, \tilde{B}, \tilde{C}, \tilde{Q}, \tilde{N})$ instead of $(A, B, C, Q, N)$ on the right-hand sides. We then have the following:

(a) $\tilde{Q}$ and $\tilde{N}$ satisfy the respective Lyapunov equations given in (3.5).

(b) The matrices $\tilde{Q}$ and $\tilde{N}$ are self-adjoint and invertible.

(c) $\tilde{u}(x, t)=v(x, t)$ and $\tilde{v}(x, t)=u(x, t)$.

Proof. Using (2.1) and (3.18), it can directly be verified that (3.5) is satisfied. The selfadjointness and invertibility of $\tilde{Q}$ and $\tilde{N}$ follow from (3.18) because $Q$ and $N$ have those properties, as stated in theorem 2.2 (ii) and (iv); hence, (b) holds. Finally, (c) is proved by direct substitution in (2.3) and (2.5) with the help of the self-adjointness of $Q$ and $N$.

\section{The scattering coefficients and Jost solutions}

In this section, we evaluate the Jost solutions and the corresponding scattering coefficients for the Zakharov-Shabat system (1.2) associated with the NLS equation (1.1) when the potential is given by (2.3) or (2.5). In analyzing (1.2), we will use the notation $\varphi=\left[\begin{array}{l}\varphi_{1} \\ \varphi_{2}\end{array}\right]$, where the subscripts 1 and 2 denote the first and second components. If $\varphi(\lambda, x, t)$ and $\bar{\varphi}(\lambda, x, t)$ are any two solutions to (1.2), their Wronskian $[\varphi ; \bar{\varphi}]$ is independent of $x$, where we have defined

$$
[\varphi ; \bar{\varphi}]:=\varphi\left(\lambda^{*}, x, t\right)^{\dagger} \bar{\varphi}(\lambda, x, t) .
$$

We stress that an overbar does not indicate complex conjugation.

Recall $[13,15]$ that the Jost solutions $\psi, \bar{\psi}, \phi$ and $\bar{\phi}$ are defined as those vector solutions to (1.2) with asymptotics

$$
\begin{aligned}
& \psi(\lambda, x, t)=\left[\begin{array}{c}
0 \\
\mathrm{e}^{\mathrm{i} \lambda x}
\end{array}\right][1+o(1)], \quad \bar{\psi}(\lambda, x, t)=\left[\begin{array}{c}
\mathrm{e}^{-\mathrm{i} \lambda x} \\
0
\end{array}\right][1+o(1)], \quad x \rightarrow+\infty, \\
& \phi(\lambda, x, t)=\left[\begin{array}{c}
\mathrm{e}^{-\mathrm{i} \lambda x} \\
0
\end{array}\right][1+o(1)], \quad \bar{\phi}(\lambda, x, t)=\left[\begin{array}{c}
0 \\
\mathrm{e}^{\mathrm{i} \lambda x}
\end{array}\right][1+o(1)], \quad x \rightarrow-\infty .
\end{aligned}
$$

When $u(\cdot, t)$ is integrable for each fixed $t$, the four Jost solutions exist and their asymptotics at the other end of the real axis yield the scattering coefficients $R, L, T_{1}, T_{\mathrm{r}}$ via 


$$
\begin{aligned}
& \psi(\lambda, x, t)=\left[\begin{array}{c}
L T_{1}^{-1} \mathrm{e}^{-\mathrm{i} \lambda x} \\
T_{1}^{-1} \mathrm{e}^{\mathrm{i} \lambda x}
\end{array}\right][1+o(1)], \quad x \rightarrow-\infty, \\
& \bar{\psi}(\lambda, x, t)=\left[\begin{array}{c}
\left(T_{\mathrm{r}}^{\dagger}\right)^{-1} \mathrm{e}^{-\mathrm{i} \lambda x} \\
-L^{\dagger}\left(T_{\mathrm{r}}^{\dagger}\right)^{-1} \mathrm{e}^{\mathrm{i} \lambda x}
\end{array}\right][1+o(1)], \quad x \rightarrow-\infty, \\
& \phi(\lambda, x, t)=\left[\begin{array}{l}
T_{\mathrm{r}}^{-1} \mathrm{e}^{-\mathrm{i} \lambda x} \\
R T_{\mathrm{r}}^{-1} \mathrm{e}^{\mathrm{i} \lambda x}
\end{array}\right][1+o(1)], \quad x \rightarrow+\infty, \\
& \bar{\phi}(\lambda, x, t)=\left[\begin{array}{c}
-R^{\dagger}\left(T_{1}^{\dagger}\right)^{-1} \mathrm{e}^{-\mathrm{i} \lambda x} \\
\left(T_{1}^{\dagger}\right)^{-1} \mathrm{e}^{\mathrm{i} \lambda x}
\end{array}\right][1+o(1)], \quad x \rightarrow+\infty .
\end{aligned}
$$

The scattering coefficients can equivalently be obtained with the help of the Wronskian defined in (4.1); in fact, we have

$$
L T_{1}^{-1}=\left(R T_{\mathrm{r}}^{-1}\right)^{\dagger}=[\phi ; \psi], \quad T_{1}^{-1}=[\bar{\phi} ; \psi], \quad T_{\mathrm{r}}^{-1}=[\bar{\psi} ; \phi] .
$$

For the Zakharov-Shabat system (1.2) we have $T_{1}=T_{\mathrm{r}}$ if the scalar potential $u(x, t)$ vanishes as $x \rightarrow \pm \infty$. However, we will retain the separate notations for $T_{l}$ and $T_{r}$ for a subsequent generalization to the matrix case, where $T_{l}$ and $T_{r}$ may differ.

The Jost solutions $\psi$ and $\bar{\psi}$ and the potential $u(x, t)$ are recovered $[13,15]$ as

$$
\begin{aligned}
& \psi(\lambda, x, t)=\left[\begin{array}{c}
0 \\
\mathrm{e}^{\mathrm{i} \lambda x}
\end{array}\right]+\int_{x}^{\infty} \mathrm{d} y K(x, y, t) \mathrm{e}^{\mathrm{i} \lambda y}, \\
& \bar{\psi}(\lambda, x, t)=\left[\begin{array}{c}
\mathrm{e}^{-\mathrm{i} \lambda x} \\
0
\end{array}\right]+\int_{x}^{\infty} \mathrm{d} y \bar{K}(x, y, t) \mathrm{e}^{-\mathrm{i} \lambda y}, \\
& u(x, t)=-2\left[\begin{array}{ll}
1 & 0
\end{array}\right] K(x, x, t)=2 \bar{K}(x, x, t)^{\dagger}\left[\begin{array}{l}
0 \\
1
\end{array}\right],
\end{aligned}
$$

from the solutions to the Marchenko integral equations

$$
\begin{aligned}
& \bar{K}(x, y, t)+\left[\begin{array}{c}
0 \\
\Omega_{1}(x+y, t)
\end{array}\right]+\int_{x}^{\infty} \mathrm{d} y K(x, z, t) \Omega_{1}(z+y, t)=\left[\begin{array}{l}
0 \\
0
\end{array}\right], \quad y>x, \\
& K(x, y, t)-\left[\begin{array}{c}
\Omega_{1}(x+y, t)^{\dagger} \\
0
\end{array}\right]-\int_{x}^{\infty} \mathrm{d} y \bar{K}(x, z, t) \Omega_{1}(z+y, t)^{\dagger}=\left[\begin{array}{l}
0 \\
0
\end{array}\right], \quad y>x .
\end{aligned}
$$

The Jost solutions $\psi$ and $\bar{\psi}$ corresponding to $u(x, t)$ given in (2.3) with $(A, B, C)=\left(A_{1}, B_{1}, C_{1}\right)$ can be evaluated by solving (4.7) and (4.8) with

$$
\Omega_{1}(y, t)=C_{1} \mathrm{e}^{-A_{1} y-4 \mathrm{i} A_{1}^{2} t} B_{1}
$$

Since

$$
\Omega_{1}(x+y, t)=C_{1} \mathrm{e}^{-A_{1} x} \mathrm{e}^{-A_{1} y-4 \mathrm{i} A_{1}^{2} t} B_{1}
$$

the Marchenko equations in (4.7) and (4.8) have integral kernels separable in $z$ and $y$ and hence they can be solved explicitly by algebraic methods, yielding

$$
\psi(\lambda, x, t)=\left[\begin{array}{c}
\mathrm{i} B_{1}^{\dagger} F_{1}(x, t)^{-1}\left(\lambda I+\mathrm{i} A_{1}^{\dagger}\right)^{-1} \mathrm{e}^{\mathrm{i} \lambda x} C_{1}^{\dagger} \\
\mathrm{e}^{\mathrm{i} \lambda x}-\mathrm{i} C_{1}\left[F_{1}(x, t)^{\dagger}\right]^{-1} N_{1} \mathrm{e}^{-2 A_{1}^{\dagger} x+4 \mathrm{i}\left(A_{1}^{\dagger}\right)^{2} t}\left(\lambda I+\mathrm{i} A_{1}^{\dagger}\right)^{-1} \mathrm{e}^{\mathrm{i} \lambda x} C_{1}^{\dagger}
\end{array}\right],
$$

10 
$\bar{\psi}(\lambda, x, t)=\left[\begin{array}{c}\mathrm{e}^{-\mathrm{i} \lambda x}+\mathrm{i} B_{1}^{\dagger} \mathrm{e}^{-2 A_{1}^{\dagger} x+4 \mathrm{i}\left(A_{1}^{\dagger}\right)^{2} t} Q_{1}\left[F_{1}(x, t)^{\dagger}\right]^{-1}\left(\lambda I-\mathrm{i} A_{1}\right)^{-1} \mathrm{e}^{-\mathrm{i} \lambda x} B_{1} \\ \mathrm{i} C_{1}\left[F_{1}(x, t)^{\dagger}\right]^{-1}\left(\lambda I-\mathrm{i} A_{1}\right)^{-1} \mathrm{e}^{-\mathrm{i} \lambda x} B_{1}\end{array}\right]$.

Similarly, the Jost solutions $\phi$ and $\bar{\phi}$ and the potential $v(x, t)$, in the form given in (2.5), are recovered as

$$
\begin{aligned}
& \phi(\lambda, x, t)=\left[\begin{array}{c}
\mathrm{e}^{-\mathrm{i} \lambda x} \\
0
\end{array}\right]+\int_{-\infty}^{x} \mathrm{~d} y M(x, y, t) \mathrm{e}^{-\mathrm{i} \lambda y} \\
& \bar{\phi}(\lambda, x, t)=\left[\begin{array}{c}
0 \\
\mathrm{e}^{\mathrm{i} \lambda x}
\end{array}\right]+\int_{-\infty}^{x} \mathrm{~d} y \bar{M}(x, y, t) \mathrm{e}^{\mathrm{i} \lambda y} \\
& v(x, t)=2\left[\begin{array}{ll}
1 & 0
\end{array}\right] \bar{M}(x, x, t)=-2 M(x, x, t)^{\dagger}\left[\begin{array}{l}
0 \\
1
\end{array}\right],
\end{aligned}
$$

from the solutions to the Marchenko integral equations

$$
\begin{aligned}
& \bar{M}(x, y, t)+\left[\begin{array}{c}
\Omega_{\mathrm{r}}(x+y, t) \\
0
\end{array}\right]+\int_{-\infty}^{x} \mathrm{~d} y M(x, z, t) \Omega_{\mathrm{r}}(z+y, t)=\left[\begin{array}{l}
0 \\
0
\end{array}\right], \quad y<x, \\
& M(x, y, t)-\left[\begin{array}{c}
0 \\
\Omega_{\mathrm{r}}(x+y, t)^{\dagger}
\end{array}\right]-\int_{-\infty}^{x} \mathrm{~d} y \bar{M}(x, z, t) \Omega_{\mathrm{r}}(z+y, t)^{\dagger}=\left[\begin{array}{l}
0 \\
0
\end{array}\right], \quad y<x .
\end{aligned}
$$

Corresponding to the potential $v(x, t)$ given in (2.5) with $(A, B, C)=\left(A_{\mathrm{r}}, B_{\mathrm{r}}, C_{\mathrm{r}}\right)$ we have

$$
\Omega_{\mathrm{r}}(y, t)=C_{\mathrm{r}} \mathrm{e}^{A_{\mathrm{r}} y+4 \mathrm{i} A_{\mathrm{r}}^{2} t} B_{\mathrm{r}},
$$

which yields an integral kernel separable in $z$ and $y$ in (4.11) and (4.12) and hence allows us to solve those integral equations explicitly by algebraic methods, yielding

$$
\begin{aligned}
& \phi(\lambda, x, t)=\left[\begin{array}{c}
\mathrm{e}^{-\mathrm{i} \lambda x}-\mathrm{i} C_{\mathrm{r}} G_{\mathrm{r}}(x, t)^{-1} N_{\mathrm{r}} \mathrm{e}^{2 A_{\mathrm{r}}^{\dagger} x-4 \mathrm{i}\left(A_{\mathrm{r}}^{\dagger}\right)^{2} t}\left(\lambda I+\mathrm{i} A_{\mathrm{r}}^{\dagger}\right)^{-1} \mathrm{e}^{-\mathrm{i} \lambda x} C_{\mathrm{r}}^{\dagger} \\
\mathrm{i} B_{\mathrm{r}}^{\dagger}\left[G_{\mathrm{r}}(x, t)^{\dagger}\right]^{-1}\left(\lambda I+\mathrm{i} A_{\mathrm{r}}^{\dagger}\right)^{-1} \mathrm{e}^{-\mathrm{i} \lambda x} C_{\mathrm{r}}^{\dagger}
\end{array}\right], \\
& \bar{\phi}(\lambda, x, t)=\left[\begin{array}{c}
\mathrm{i} C_{\mathrm{r}} G_{\mathrm{r}}(x, t)^{-1}\left(\lambda I-\mathrm{i} A_{\mathrm{r}}\right)^{-1} \mathrm{e}^{\mathrm{i} \lambda x} B_{\mathrm{r}} \\
\mathrm{e}^{\mathrm{i} \lambda x}+\mathrm{i} B_{\mathrm{r}}^{\dagger}\left[G_{\mathrm{r}}(x, t)^{\dagger}\right]^{-1} Q_{\mathrm{r}} \mathrm{e}^{2 A_{\mathrm{r}} x+4 \mathrm{i} A_{\mathrm{r}}^{2} t}\left(\lambda I-\mathrm{i} A_{\mathrm{r}}\right)^{-1} \mathrm{e}^{\mathrm{i} \lambda x} B_{\mathrm{r}}
\end{array}\right] .
\end{aligned}
$$

Proposition 4.1. Assume that the triplet $(A, B, C)$ corresponds to a minimal realization in (2.12) and that all eigenvalues of $A$ have positive real parts. Let $(Q, N)$ correspond to the unique solution to the Lyapunov system (2.1) and let $(F, G)$ be as in (2.2) and (2.4). We then have the following:

(a) $F(x, t)^{-1} \rightarrow 0$ and $G(x, t)^{-1} \rightarrow 0$ as $x \rightarrow \pm \infty$.

(b) $\mathrm{e}^{-2 A^{\dagger} x+4 \mathrm{i}\left(A^{\dagger}\right)^{2} t} Q\left[F(x, t)^{\dagger}\right]^{-1} \rightarrow N^{-1}$ as $x \rightarrow-\infty$.

(c) $\left[F(x, t)^{\dagger}\right]^{-1} N \mathrm{e}^{-2 A^{\dagger} x+4 i\left(A^{\dagger}\right)^{2} t} \rightarrow Q^{-1}$ as $x \rightarrow-\infty$.

(d) $\left[G(x, t)^{\dagger}\right]^{-1} Q \mathrm{e}^{2 A x+4 i A^{2} t} \rightarrow N^{-1}$ as $x \rightarrow+\infty$.

(e) $G(x, t)^{-1} N \mathrm{e}^{2 A^{\dagger} x-4 \mathrm{i}\left(A^{\dagger}\right)^{2} t} \rightarrow Q^{-1}$ as $x \rightarrow+\infty$.

Proof. The proofs involving $G$ are obtained by using (2.6) the same way as in the proofs for $F$, and hence we will only give the proofs for $F$. Using (2.2) and (2.7) we see that

$$
F=\mathrm{e}^{\beta^{\dagger}}+Q \mathrm{e}^{-\beta} N, \quad F^{\dagger}=\mathrm{e}^{\beta}+N \mathrm{e}^{-\beta^{\dagger}} Q,
$$


and hence

$F=\mathrm{e}^{\beta^{\dagger}}\left[I+\mathrm{e}^{-\beta^{\dagger}} Q \mathrm{e}^{-\beta} N\right], \quad Q^{-1} F N^{-1} \mathrm{e}^{\beta}=I+Q^{-1} \mathrm{e}^{\beta^{\dagger}} N^{-1} \mathrm{e}^{\beta}$,

$F^{\dagger} Q^{-1} \mathrm{e}^{\beta^{\dagger}} N^{-1}=\left[I+\mathrm{e}^{\beta} Q^{-1} \mathrm{e}^{\beta^{\dagger}} N^{-1}\right], \quad \mathrm{e}^{\beta^{\dagger}} N^{-1} F^{\dagger}=\left[I+\mathrm{e}^{\beta^{\dagger}} N^{-1} \mathrm{e}^{\beta} Q^{-1}\right] Q$.

Forming the inverses from (4.13) and (4.14) we obtain

$$
\begin{aligned}
& F^{-1}=\left[I+\mathrm{e}^{-\beta^{\dagger}} Q \mathrm{e}^{-\beta} N\right]^{-1} \mathrm{e}^{-\beta^{\dagger}}, \quad F^{-1}=N^{-1} \mathrm{e}^{\beta}\left[I+Q^{-1} \mathrm{e}^{\beta^{\dagger}} N^{-1} \mathrm{e}^{\beta}\right]^{-1} Q^{-1}, \\
& \mathrm{e}^{-\beta^{\dagger}} Q\left(F^{\dagger}\right)^{-1}=N^{-1}\left[I+\mathrm{e}^{\beta} Q^{-1} e^{\beta^{\dagger}} N^{-1}\right]^{-1}, \\
& \left(F^{\dagger}\right)^{-1} N \mathrm{e}^{-\beta^{\dagger}}=Q^{-1}\left[I+\mathrm{e}^{\beta^{\dagger}} N^{-1} \mathrm{e}^{\beta} Q^{-1}\right]^{-1} .
\end{aligned}
$$

By letting $x \rightarrow \pm \infty$ in (4.15) we prove (a). By letting $x \rightarrow-\infty$ in (4.16) and (4.17) we prove (b) and (c), respectively. The remaining proofs involving $G$ are obtained in a similar manner.

The results in the following two propositions are needed in proving theorem 4.4, and we refer the reader to $[5,9]$ for such results and their proofs.

Proposition 4.2. For any triplet of matrices $(A, B, C)$ with sizes $p \times p, p \times n$ and $n \times p$, respectively, we have

$$
\left[I_{n}+C\left(\lambda I_{p}-A\right)^{-1} B\right]^{-1}=I_{n}-C\left(\lambda I_{p}-A+B C\right)^{-1} B .
$$

Proposition 4.3. For any matrices $U$ and $V$ with sizes $n \times p$ and $p \times n$, respectively, we have the determinant identity

$$
\operatorname{det}\left(I_{n}+U V\right)=\operatorname{det}\left(I_{p}+V U\right)
$$

Theorem 4.4. Assume that the triplet $(A, B, C)$ corresponds to a minimal realization in (2.12) and that all eigenvalues of $A$ have positive real parts. Let $(Q, N)$ correspond to the unique solution to the Lyapunov system in (2.1), (F,u) be as in (2.2) and (2.3) and the scattering coefficients be defined as in (4.2)-(4.5). We then have the following:

(a) $u(x, t) \rightarrow 0$ as $x \rightarrow \pm \infty$.

(b) For $\lambda \in \mathbf{R}$, the transmission coefficients $T_{1}$ and $T_{\mathrm{r}}$ appearing in (4.2) and (4.4), respectively, and their inverses are given by

$$
\begin{aligned}
& T_{1}^{-1}=1-\mathrm{i} C Q^{-1}\left(\lambda I+\mathrm{i} A^{\dagger}\right)^{-1} C^{\dagger}, \\
& T_{1}=1+\mathrm{i} C(\lambda I-\mathrm{i} A)^{-1} Q^{-1} C^{\dagger}, \\
& T_{\mathrm{r}}^{-1}=1-\mathrm{i} B^{\dagger}\left(\lambda I+\mathrm{i} A^{\dagger}\right)^{-1} N^{-1} B, \\
& T_{\mathrm{r}}=1+\mathrm{i} B^{\dagger} N^{-1}(\lambda I-\mathrm{i} A)^{-1} B,
\end{aligned}
$$

and hence they are functions of $\lambda$ alone and do not depend on $t$.

(c) The reflection coefficients $L(\lambda, t)$ and $R(\lambda, t)$ appearing in (4.2) and (4.4) are both identically zero.

(d) The transmission coefficients can be written as the ratio of two determinants as

$$
T_{1}(\lambda)=\frac{\operatorname{det}\left(\lambda I+\mathrm{i} A^{\dagger}\right)}{\operatorname{det}(\lambda I-\mathrm{i} A)}, \quad T_{\mathrm{r}}(\lambda)=\frac{\operatorname{det}\left(\lambda I+\mathrm{i} A^{\dagger}\right)}{\operatorname{det}(\lambda I-\mathrm{i} A)} .
$$


Proof. Let us compare (4.2) and (4.3) with (4.9) and (4.10) by ignoring the subscript 1 in (4.9) and (4.10). Using proposition 4.1 (a) in the first component of (4.9), we see that $L T_{1}^{-1}=0$. Then, with the help of (4.6), we also conclude $R T_{\mathrm{r}}^{-1}=0$. Using proposition 4.1 (c) in the second component of (4.9) we obtain (4.18), and similarly by using proposition 4.1 (b) in the first component in (4.10) we obtain

$$
\left(T_{\mathrm{r}}^{-1}\right)^{\dagger}=1+\mathrm{i} B^{\dagger} N^{-1}(\lambda I-\mathrm{i} A)^{-1} B .
$$

Applying proposition 4.2 on (4.18) and (4.20) and simplifying the resulting expressions with the help of (2.1), we obtain (4.19) and (4.21). We then get (c) from $L T_{1}^{-1}=0$ and $R T_{\mathrm{r}}^{-1}=0$. Applying proposition 4.3 onto (4.19) and (4.21) and using (2.1) we obtain (4.22).

\section{An example}

We conclude our paper by providing an application of theorem 3.2 to a specific case. In example 7.2 of [3] we evaluated the exact solution to the NLS equation corresponding to the triplet $(A, B, C)$ with

$$
A=\left[\begin{array}{cc}
2 & 0 \\
0 & -1
\end{array}\right], \quad B=\left[\begin{array}{l}
1 \\
1
\end{array}\right], \quad C=\left[\begin{array}{ll}
1 & -1
\end{array}\right] .
$$

We incorrectly conjectured in that example that we had a nonsoliton solution because one of the eigenvalues of $A$ was not positive. Using the result of theorem 3.2 of the present paper, we are now able to confirm that that solution is indeed a two-soliton solution. For this, we proceed as follows.

Using the triplet $(A, B, C)$ of (5.1), we solve (2.1) in a straightforward manner and get

$$
Q=\left[\begin{array}{cc}
1 / 4 & -1 \\
-1 & -1 / 2
\end{array}\right], \quad N=\left[\begin{array}{cc}
1 / 4 & 1 \\
1 & -1 / 2
\end{array}\right]
$$

We then construct $(\tilde{A}, \tilde{B}, \tilde{C}, \tilde{Q}, \tilde{N}, \tilde{F})$ via (3.9)-(3.13) and obtain

$$
\begin{aligned}
\tilde{A} & =\left[\begin{array}{ll}
2 & 0 \\
0 & 1
\end{array}\right], \quad \tilde{B}=\left[\begin{array}{l}
3 \\
2
\end{array}\right], \quad \tilde{C}=\left[\begin{array}{ll}
3 & -2
\end{array}\right], \\
\tilde{Q} & =\left[\begin{array}{cc}
9 / 4 & -2 \\
-2 & 2
\end{array}\right], \quad \tilde{N}=\left[\begin{array}{cc}
9 / 4 & 2 \\
2 & 2
\end{array}\right], \\
\tilde{F} & =\left[\begin{array}{cc}
\mathrm{e}^{4 x-16 \mathrm{i} t}+\frac{81}{16} \mathrm{e}^{-4 x-16 \mathrm{i} t}-4 \mathrm{e}^{-2 x-4 \mathrm{i} t} & \frac{9}{2} \mathrm{e}^{-4 x-16 \mathrm{i} t}-4 \mathrm{e}^{-2 x-4 \mathrm{i} t} \\
-\frac{9}{2} \mathrm{e}^{-4 x-16 \mathrm{i} t}+4 \mathrm{e}^{-2 x-4 \mathrm{i} t} & \mathrm{e}^{2 x-4 \mathrm{i} t}-4 \mathrm{e}^{-4 x-16 \mathrm{i} t}+4 \mathrm{e}^{-2 x-4 \mathrm{i} t}
\end{array}\right] .
\end{aligned}
$$

As seen from (5.2), the eigenvalue $(-1)$ of $A$ is transformed into the eigenvalue $(+1)$ of $\tilde{A}$. The potential $\tilde{u}(x, t)$, or equivalently $u(x, t)$, is then constructed via (2.3) and we get

$$
u(x, t)=\frac{8 \mathrm{e}^{4 \mathrm{i} t}\left(9 \mathrm{e}^{-4 x}+16 \mathrm{e}^{4 x}\right)-32 \mathrm{e}^{16 i t}\left(4 \mathrm{e}^{-2 x}+9 \mathrm{e}^{2 x}\right)}{-128 \cos (12 t)+4 \mathrm{e}^{-6 x}+16 \mathrm{e}^{6 x}+81 \mathrm{e}^{-2 x}+64 \mathrm{e}^{2 x}},
$$

agreeing with the potential of example 7.2 of [3]. For this potential, the transmission coefficients are evaluated via (4.22) as

$$
T_{1}(\lambda)=T_{\mathrm{r}}(\lambda)=\frac{(\lambda+2 \mathrm{i})(\lambda+\mathrm{i})}{(\lambda-2 \mathrm{i})(\lambda-\mathrm{i})},
$$

because the real parts of all eigenvalues of $\tilde{A}$ in the associated triplet $(\tilde{A}, \tilde{B}, \tilde{C})$ are positive. As for the norming constants associated with the bound states at $\lambda=2 \mathrm{i}$ and $\lambda=\mathrm{i}$, we need 
to transform the triplet $(\tilde{A}, \tilde{B}, \tilde{C})$ of (5.2) into another triplet $(A, B, C)$, different from (5.1), so that we will have $B=\left[\begin{array}{l}1 \\ 1\end{array}\right]$. By using (2.16) we obtain

$M=\left[\begin{array}{ll}1 & 0 \\ 0 & 1\end{array}\right], \quad S=\left[\begin{array}{ll}3 & 0 \\ 0 & 2\end{array}\right], \quad A=\left[\begin{array}{ll}2 & 0 \\ 0 & 1\end{array}\right], \quad B=\left[\begin{array}{l}1 \\ 1\end{array}\right], \quad C=\left[\begin{array}{ll}9 & -4\end{array}\right]$.

Thus, the norming constant at $\lambda=2 \mathrm{i}$ is 9 and the norming constant at $\lambda=\mathrm{i}$ is -4 . Finally, the Jost solutions $\psi(\lambda, x, t)$ and $\bar{\psi}(\lambda, x, t)$ to the Zakharov-Shabat system (1.2) are obtained via (4.9) and (4.10), respectively, by using $(\tilde{A}, \tilde{B}, \tilde{C}, \tilde{Q}, \tilde{N}, \tilde{F})$ for $\left(\tilde{A}_{1}, \tilde{B}_{1}, \tilde{C}_{1}, \tilde{Q}_{1}, \tilde{N}_{1}, \tilde{F}_{1}\right)$ there. For example, for the Jost solution $\psi$ we get

$\psi(\lambda, x, t)=\left[\begin{array}{c}0 \\ \mathrm{e}^{\mathrm{i} \lambda x}\end{array}\right]+\frac{\left[\begin{array}{c}4 \mathrm{i}^{-4 x+4 \mathrm{it}} g_{1}(\lambda, x, t) \\ 4 \mathrm{i} g_{2}(\lambda, x, t)\end{array}\right] \mathrm{e}^{\mathrm{i} \lambda x}}{(\lambda+2 \mathrm{i})(\lambda+\mathrm{i})\left[-128 \cos (12 t)+4 \mathrm{e}^{-6 x}+16 \mathrm{e}^{6 x}+81 \mathrm{e}^{-2 x}+64 \mathrm{e}^{2 x}\right]}$,

where we have defined

$g_{1}(\lambda, x, t):=36(\lambda+\mathrm{i}) \mathrm{e}^{6 x+12 \mathrm{i} t}+16(\lambda-\mathrm{i}) \mathrm{e}^{2 x+12 \mathrm{i} t}-16(\lambda+2 \mathrm{i}) \mathrm{e}^{8 x}-9(\lambda-2 \mathrm{i})$,

$g_{2}(\lambda, x, t):=48(\lambda+\mathrm{i}) \mathrm{e}^{12 \mathrm{i} t}+48(\lambda+2 \mathrm{i}) \mathrm{e}^{-12 i t}-6 \lambda \mathrm{e}^{-6 x}-81(\lambda+\mathrm{i}) \mathrm{e}^{-2 x}-32(\lambda+2 \mathrm{i}) \mathrm{e}^{2 x}$.

\section{Acknowledgments}

One of the authors (TA) is greatly indebted to the University of Cagliari for its hospitality during a recent visit. The research leading to this paper was supported in part by the National Science Foundation under grant DMS-0610494, INdAM and the Autonomous Region of Sardinia.

\section{References}

[1] Ablowitz M J and Clarkson P A 1991 Solitons, Nonlinear Evolution Equations and Inverse Scattering (Cambridge: Cambridge University Press)

[2] Ablowitz M J and Segur H 1981 Solitons and the Inverse Scattering Transform (Philadelphia: SIAM)

[3] Aktosun T, Demontis F and van der Mee C 2007 Exact solutions to the focusing nonlinear Schrödinger equation Inverse Problems 23 2171-95

[4] Aktosun T and van der Mee C 2006 Explicit solutions to the Korteweg-de Vries equation on the half-line Inverse Problems 22 2165-74

[5] Bart H, Gohberg I and Kaashoek M A 1979 Minimal Factorization of Matrix and Operator Functions (Basel: Birkhäuser)

[6] Demontis F 2007 Direct and inverse scattering of the matrix Zakharov-Shabat system PhD Thesis University of Cagliari, Italy

[7] Demontis F and van der Mee C 2008 Marchenko equations and norming constants of the matrix Zakharov-Shabat system Oper. Matrices 2 79-113

[8] Demontis F and van der Mee C 2008 Explicit solutions of the cubic matrix nonlinear Schrödinger equation Inverse Problems $240252016 \mathrm{pp}$

[9] Dym H 2007 Linear Algebra in Action (Providence, RI: American Mathematical Society)

[10] Hasegawa A and Matsumoto M 2002 Optical Solitons in Fibers 3rd edn (Berlin: Springer)

[11] Hasegawa A and Tappert F 1973 Transmission of stationary nonlinear optical pulses in dispersive dielectric fibers: I. Anomalous dispersion Appl. Phys. Lett. 23 142-4

[12] Hasegawa A and Tappert F 1973 Transmission of stationary nonlinear optical pulses in dispersive dielectric fibers: II. Normal dispersion Appl. Phys. Lett. 23 171-2

[13] Novikov S, Manakov S V, Pitaevskii L P and Zakharov V E 1984 Theory of Solitons (New York: Consultants Bureau)

[14] Zakharov V E 1968 Stability of periodic waves of finite amplitude on the surface of a deep fluid J. Appl. Mech. Tech. Phys. 4 190-4

[15] Zakharov V E and Shabat A B 1972 Exact theory of two-dimensional self-focusing and one dimensional self-modulation of waves in nonlinear media Sov. Phys. JETP 34 62-9 